\title{
piRNAs, master regulators of gene expression
}

Cell Research (2014) 24:779-780. doi:10.1038/cr.2014.78; published online 20 June 2014

Piwi-interacting RNAs (piRNAs) have a major function in the repression of transposable elements in the germline; in addition, they have been proposed to regulate gene expression. A recent study in Cell Research reveals a general role for piRNAs in the massive mRNA decay during mouse spermiogenesis, reinforcing this emerging function of piRNAs.

piRNAs form a distinct class of small non-coding RNAs that are associated with specific Argonaute proteins, the Piwi proteins. Genetic studies have shown that the Piwi/piRNA pathway has an evolutionarily conserved role in the silencing of transposable elements (TE) in the germline of animals. A large proportion of piRNAs indeed derive from $\mathrm{TE}$ sequences and target complementary sequences, inducing TE silencing at transcriptional or post-transcriptional levels [1]. However, piRNAs are not systematically produced from TE sequences, and they can be generated from genomic clusters unrelated to TE. This is the case in C. elegans germ cells and during late spermatogenesis in mice. The existence of piRNAs unrelated to TE suggests that they have additional functions independent of TE silencing, such as potential roles in the regulation of gene expression.

The first hint of a role for piRNAs in cellular mRNA regulation came from a study in the Drosophila embryo [2]. Nanos, an essential posterior determinant required for abdomen and germline development in the embryo, is expressed as a gradient arising from the posterior pole. Translational repres- sion of large amounts of nanos mRNA present in the non-posterior part of the embryo is necessary for normal development of the anterior structures. Translational repression and decay of nanos mRNA involves deadenylation by the CCR4-NOT deadenylation complex and Smaug, an RNA binding protein that binds to nanos 3'UTR and recruits the deadenylation complex. A candidate approach was used to screen for additional genes involved in deadenylation of unlocalized nanos mRNA and identified components of the piRNA pathway. Specifically, the cytoplasmic Piwi proteins Aubergine and Argonaute 3 were found in complex with Smaug and the CCR4 deadenylase in early embryos, and Aubergine associated with nanos mRNA. Importantly, piRNAs that target the nanos 3'UTR with imperfect complementarity were functionally validated to be required for nanos mRNA deadenylation and normal antero-posterior embryonic patterning [2]. These data pioneered the notion that piRNAs could play a vital role in gene regulation, via a mechanism similar to that used by microRNAs, which involves imperfect base pairing to target $\mathrm{mR}$ NAs and the recruitment of the CCR4NOT deadenylation complex.

More recently, several studies in C. elegans have reported a widespread targeting of cellular mRNAs by piRNAs. As mentioned above, piRNAs (21U-RNAs) in C. elegans do not derive from TE sequences. These 21U-RNAs are loaded into the Argonaute protein PRG-1 and target mRNAs through imperfect complementarity. In turn, PRG-1 association with mRNAs induces the production of secondary small RNAs, called 22G, which are antisense to PRG-1 targets $[3,4]$. Because these secondary $22 \mathrm{G}-\mathrm{RNAs}$ are perfectly complementary to mRNAs, they allow to accurately identify mRNAs that are targeted by piRNAs. 22G-RNAs are then loaded into Argonaute proteins of either the WAGO type which induces target repression [3, 4], or CSR-1 which prevents this repression and/or activates expression $[5,6]$. This phenomenon is involved in the recognition of expressed transcripts (self) versus silenced transcripts/foreign sequences (non-self) in the germline across generations. It also implies a genome-wide scanning and targeting of germline mRNAs by piRNAs, which is made possible by the diversity of piRNAs and their association with target mRNAs through partial base pairing. These studies provide compelling evidence that piRNAs play a key role in controlling gene expression in $C$. elegans germ cells.

The new study by Gou et al. [7] adds to the picture a novel example of a widespread role for piRNAs in gene regulation in the mouse, strongly reinforcing the general aspect of this function of piRNAs. The Piwi proteins Mili, Miwi and Miwi2 are essential for spermatogenesis in mice. Here, piRNAs are produced in two waves; the first occurs during early spermatogenesis in fetal and perinatal stages, generating piRNAs enriched in TE sequences, while the second occurs during postnatal spermatogenesis and produces piRNAs unrelated to TE sequences, the so-called pachytene 
piRNAs, that are coexpressed with and loaded into Miwi [8]. As pachytene piRNAs do not show strong complementarity to any genomic sequence, except the loci that produce them, their function has remained a mystery.

One previous study has reported the direct interaction of Miwi with a set of cellular mRNAs and its role in their stabilization [9]. These mRNAs are stored in repressed mRNPs in post-meiotic round spermatids and translated thereafter to participate in spermiogenesis, a process required for sperm maturation. Miwi association was reported to be independent of piRNAs in this particular case, given that Miwi-associated mRNAs and piRNAs did not cosediment in density gradients.

Gou et al. [7] address Miwi function in elongating spermatids, a later stage of spermatogenesis. Miwi was found to associate with more than 7 000 cellular mRNAs, among which about $60 \%$ were upregulated upon Miwi knockdown, indicating a role for Miwi in mRNA destabilization at this stage. The authors then searched for low complementarity between Miwi-loaded pachytene piRNAs and 3'UTR of Miwi-associated mRNAs using miRanda, a software for predicting microRNA targets. Perharps not unexpectedly, considering the loose base pairing requested and the high complexity of piRNAs, prominent potential targeting was identified. Nonetheless, this targeting could be validated in a number of cases using either luciferase reporter assays in a spermatocyte cell line, or more importantly, electroporation into adult mouse testes of antisense RNAs against the two most abundant piRNAs, which resulted in elevated expression of the corresponding target mRNAs. In addition, the global levels of mRNA expression inversely correlated with the number of predicted piRNA target sites in these mRNAs and the abundance of the targeting piRNAs, lend- ing support to the role of piRNAs in mRNA destabilization.

Significantly, the mechanism of piRNA-mediated mRNA destabilization was also addressed and involved deadenylation by CAF1, the second deadenylase of the CCR4-NOT deadenylation complex, in a process similar to that used in the Drosophila embryo for piRNA-dependent mRNA decay (although the specific protein interactions might be different between species). Miwi associated directly with CAF1, and mRNAs upregulated upon CAF1 knockdown largely overlapped $(90 \%)$ with those upregulated upon Miwi knockdown.

Investigation of the temporal assembly of a piRNA-dependent repressor complex nicely reconciled both functions of Miwi in mRNA stabilization and decay, since Miwi-CAF1 interaction, and the levels of piRNAs in complex with CAF1 were shown to peak in elongating spermatids. This switch in Miwi function also correlated with a progressive increase in Miwi loading with piRNAs [10]. Therefore, Miwi appears to be involved first in spermiogenic mRNA stabilization in round spermatids, possibly in an unloaded form, and subsequently, in its loaded form, in a general mRNA decay program in elongating spermatids through its interaction with CAF1.

A close parallel in function and mechanism of action exists between Aubergine in early Drosophila embryogenesis and Miwi during sperm maturation, two developmental processes where massive mRNA degradation takes place. The tight temporal regulation required to ensure the correct timing of mRNA decay in spermatids is likely to involve additional factors, such as Smaug in the Drosophila embryo.

These data highlight key functions of Miwi and pachytene piRNAs in genome-wide gene regulation during spermatogenesis. They substantially broaden our understanding of the role of piRNAs in gene regulation, and strengthen the notion of a general scanning and targeting of mRNAs by piRNAs, with a potential widespread impact on gene regulation.

How general is this regulatory mechanism in different processes and species? This function of piRNAs is emerging and other examples are being reported. Specific piRNAs are involved in the repression of CREB2 in Aplysia neurons for memory formation [11]. Moreover, a new study describes yet another vital role for a female-specific piRNA in sex determination in Bombyx mori through the repression of a cellular mRNA by cleavage [12]. Thorough investigation of this "novel" role of piRNAs should bring about more exciting discoveries in the near future.

\section{Martine Simonelig ${ }^{1}$}

${ }^{l} m R N A$ Regulation and Development, Institute of Human Genetics, CNRS UPR1142, 141 rue de la Cardonille, 34396 Montpellier Cedex 5, France

Correspondence: Martine Simonelig Tel: 334343599 59; Fax: 33434359957

E-mail: Martine.Simonelig@igh.cnrs.fr

\section{References}

1 Ishizu H, Siomi H, Siomi MC. Genes Dev 2012; 26:2361-2373.

2 Rouget C, Papin C, Boureux A, et al. $\mathrm{Na}$ ture 2010; 467:1128-1132.

3 Bagijn MP, Goldstein LD, Sapetschnig A, et al. Science 2012; 337:574-578.

4 Lee HC, Gu W, Shirayama M, et al. Cell 2012; 150:78-87.

5 Seth M, Shirayama M, Gu W, et al. Dev Cell 2013; 27:656-663.

6 Wedeles CJ, Wu MZ, Claycomb JM. Dev Cell 2013; 27:664-671.

7 Gou LT, Dai P, Yang JH, et al. Cell Res 2014; 24:680-700.

8 Li XZ, Roy CK, Dong X, et al. Mol Cell 2013; 50:67-81.

9 Vourekas A, Zheng Q, Alexiou P, et al. Nat Struct Mol Biol 2012; 19:773-781.

10 Zhao S, Gou LT, Zhang M, et al. Dev Cell 2013; 24:13-25.

11 Rajasethupathy P, Antonov I, Sheridan R, et al. Cell 2012; 149:693-707.

12 Kiuchi T, Koga H, Kawamoto M, et al. $\mathrm{Na}$ ture 2014; 509:633-636. 